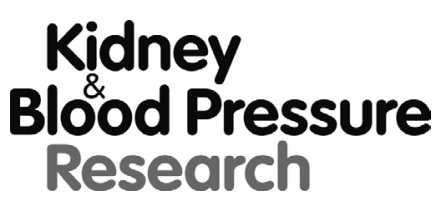

Kidney Blood Press Res 2017;42:837-843

DOI: $10.1159 / 000484426$

Published online: October 27, 2017

Accepted: August 08, 2017

This article is licensed under the Creative Commons Attribution-NonCommercial-NoDerivatives 4.0 International License (CC BY-NC-ND) (http://www.karger.com/Services/OpenAccessLicense). Usage and distribution for commercial purposes as well as any distribution of modified material requires written permission.
for

\title{
Treatment of Enterococcal Peritonitis in Peritoneal Dialysis Patients by Oral Amoxicillin or Intra-Peritoneal Vancomcyin: a Retrospective Study
}

\author{
Cheuk Chun Szeto Jack Kit-Chung Ng Kai Ming Chow Bonnie Ching-Ha Kwan \\ Vickie Wai-Ki Kwong Man-Ching Law Chi Bon Leung Philip Kam-To Li
}

Carol and Richard Yu Peritoneal Dialysis Research Centre, Department of Medicine \& Therapeutics, Prince of Wales Hospital, The Chinese University of Hong Kong, Shatin, Hong Kong SAR, China

\section{Key Words}

Renal failure $\cdot$ Infection • Survival

\begin{abstract}
Background/Aims: Enterococcal peritonitis in peritoneal dialysis (PD) patients is associated with a high complication rate. The optimal treatment regimen of PD-related enterococcal peritonitis is controversial. The latest international guideline recommends intra-peritoneal (IP) vancomycin. Although ampicillin is often effective for systemic enterococcal infections, they have little in vitro activity when added to common PD solutions. Since oral amoxicillin achieves therapeutic drug level in the peritoneal cavity, we explore the efficacy of oral amoxicillin for enterococcal peritonitis. Methods: We studied 105 episodes of enterococcal peritonitis over 20 years in our unit; 43 (41.0\%) were treated with oral amoxicillin, and 62 (59.0\%) with IP vancomycin. Their clinical outcome was reviewed. Result: The overall primary response rate to oral amoxicillin and IP vancomycin was $76.4 \%$ and $85.5 \%$, respectively $(p=0.3)$. The complete cure rate of oral amoxicillin and IP vancomycin was $55.8 \%$ and $54.8 \%$, respectively $(p=0.8)$. When the 5 episodes of ampicillin-resistant Enterococcus episodes were excluded, the primary response rate and complete cure rate of oral amoxicillin were $86.8 \%$ and $63.2 \%$, respectively. Conclusion: Oral amoxicillin has an excellent primary response rate and complete cure rate for PD-related peritonitis episodes caused by Enterococcus species, indicating that oral amoxicillin is a valid and convenient therapeutic option for enterococcal peritonitis episodes.




\section{Kidney Blood Pressure Research}

Szeto et al.: Oral Amoxicillin For Enterococcal Peritonitis

\section{Introduction}

Peritoneal dialysis (PD) is now used widely as the renal replacement therapy for patients with end stage renal disease (ESRD) [1], and is the first-line treatment of end stage renal disease (ESRD) in Hong Kong [2]. Although less than 4\% of the peritonitis episodes resulted in death, it is the major cause of technique failure and conversion to long term hemodialysis in PD patients $[3,4]$. Peritonitis soon after the initiation of PD is a particularly important predictor of mortality in elderly PD patients [5]. Most of the PD-related peritonitis episodes are caused by Staphylococcus aureus, coagulase negative staphylococcal species, Enterobacteriaceae and Pseudomonas species [4]. Enterococcal species account for less than 5\% of PD-related peritonitis [4]. However, peritonitis episodes caused by Enterococcal species are associated with particularly high rates of catheter removal, permanent hemodialysis transfer, and death [6, 7]. The treatment of PD-related enterococcal peritonitis is, unfortunately, difficult and optimal regimen controversial. Although there may be clinical response to its empirical therapy [8], treatment with first generation cephalosporin is not advisable because of intrinsic resistance to cephalosporins [9]. The latest guideline by the International Society for Peritoneal Dialysis (ISPD) recommends that peritonitis episodes caused by Enterococcal species should be treated with intra-peritoneal (IP) vancomycin unless the organism is resistant to vancomycin [10]. Although ampicillin and amoxicillin are often effective for systemic enterococcal infections, they have little in vitro activity when added to common PD solutions [11]. Since systemic administration of ampicillin and amoxicillin achieves therapeutic drug level in the peritoneal cavity $[12,13]$, it seems logical to use oral ampicillin or amoxicillin for the treatment of PD-related peritonitis caused by Enterococcus species. Here, we report the efficacy of oral amoxicillin for enterococcal peritonitis.

\section{Materials and Methods}

\section{Case selection}

The study was approved by the Clinical Research Ethics Committee of the Chinese University of Hong Kong. All procedures are in adherence to the Declaration of Helsinki. All episodes of PD peritonitis in our unit from 1996 to 2015 were reviewed. The diagnosis of peritonitis was based on at least two of the following [10]: (a) abdominal pain or cloudy peritoneal dialysis effluent (PDE); (b) leukocytosis in PDE (WBC > 100/ $\mathrm{ml}$ ); and (c) positive Gram-stain or culture from PDE. Episodes with peritoneal eosinophilia but negative bacterial culture were excluded. Exit site infection was diagnosed when there was purulent drainage, with or without erythema, from the exit site [14].

During the study period, 3194 episodes of peritonitis were recorded. Enterococcus species were isolated from the PDE in 105 episodes (3.3\%). Their case records were reviewed. Demographic characteristics, underlying medical conditions, previous peritonitis, antibiotic regimen, response to therapy, and clinical outcome were examined.

\section{Clinical Management}

During the study period, the empirical antibiotic therapy for peritonitis episodes were according to the standard antibiotic protocol of our center, which was changed systemically over time, and generally intra-peritoneal administration of cefazolin plus an aminoglycoside or ceftazidime. When Enterococcus species was identified in PDE, antibiotic therapy was immediately changed to either oral amoxicillin (500 mg thrice daily) or intermittent intra-peritoneal (IP) vancomycin (1 gram every 4 to 5 days) for 2 to 3 weeks as decided by individual nephrologist. In general, when the therapeutic response to oral amoxicillin was suboptimal after 5 days of treatment, the antibiotic would be changed to IP vancomycin. Primary response was defined as resolution of abdominal pain, clearing of dialysate, and PDE neutrophil count less than 100/ $\mathrm{ml}$ on day-10 with antibiotics alone. When the PDE did not clear up after 10 days of effective antibiotic, the PD catheter was removed, and effective antibiotics were continued for another two weeks.

All the patients were followed for 12 months after their treatment completed. Primary end points are 


\section{Kidney \\ Blood Pressure \\ Research}

primary response rate and complete cure rate. Primary response was defined as resolution of abdominal pain, clearing of dialysate, and PDE neutrophil count less than 100/ml on day-10 with antibiotics alone. Complete cure was defined as complete resolution of peritonitis by antibiotics alone without relapse or recurrence within 4 weeks of completion of therapy. Relapsing and recurrent peritonitis episodes were defined by the ISPD guideline [10].

\section{Statistical Analysis}

Statistical analysis was performed by SPSS for Windows software version 18.0 (SPSS Inc., Chicago, IL). All data were expressed in mean \pm SD unless otherwise specified. Data were compared by Chi square test, Fisher's exact test, or Student's t test as appropriate. A p value of less than 0.05 was considered significant. All probabilities were two-tailed.

\section{Results}

During the study period, 3194 episodes of PD-related peritonitis were recorded in our unit. The overall peritonitis rate was 0.47 episodes per patient-year. We identified 105 episodes of enterococcal peritonitis; 67 episodes had mixed bacterial growth, while 38 episodes were simple enterococcal peritonitis. Of the 105 episodes, 58 (55.2\%) were caused by E. faecalis, 11 (10.5\%) by E. faecium, and 36 (34.3\%) by other species. Ampicillin resistance was present in 21 isolates $(20.0 \%)$. Six patients $(5.7 \%)$ had concomitant exit site infection, but none was due to Enterococcus species.

The primary treatment of the enterococcal peritonitis episodes was oral amoxicillin and IP vancomycin in $43(41.0 \%)$ and 62 cases (59.0\%), respectively. Their demographic and baseline clinical data are summarized and compared in Table 1. In essence, there was no significant difference between patients who received oral amoxicillin and IP vancomycin, except that patients who receive oral amoxicillin as their primary treatment were more

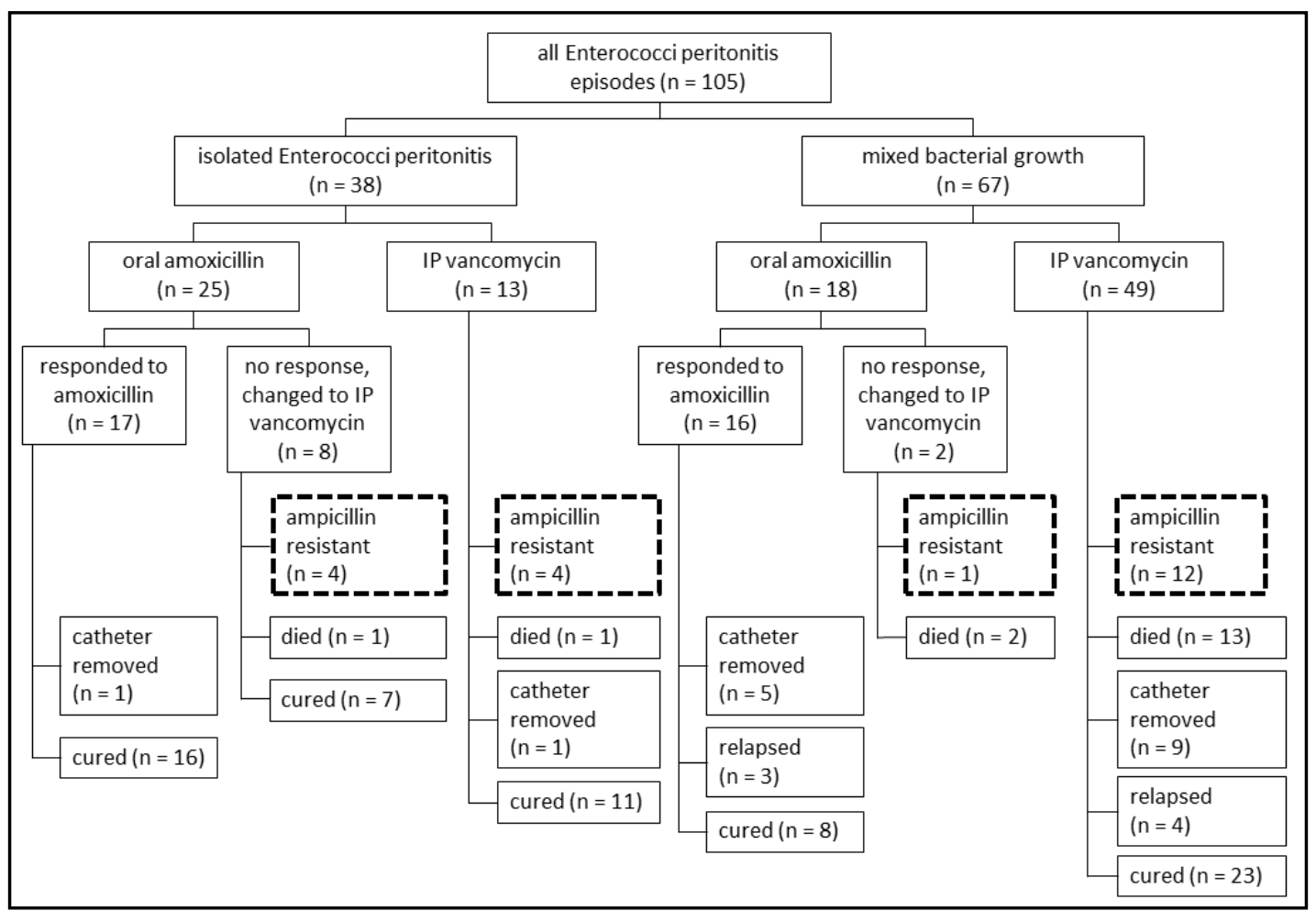

Fig. 1. Summary of clinical outcome of enterococcal peritonitis. 


\section{Kidney Bloód Pressure Research}

TABLE 1. Baseline characteristics of the patients. PD, peritoneal dialysis. *Data were compared by ${ }^{\mathrm{a}} \mathrm{Chi}$ square test, ${ }^{b}$ Student's t test, and 'Mann Whitney U test. ${ }^{* *}$ Data presented as median (inter-quartile range)

\begin{tabular}{|c|c|c|c|}
\hline Group & Oral amoxicillin & IP vancomycin & P value* \\
\hline No. of patient & 43 & 62 & \\
\hline $\operatorname{Sex}(M: F)$ & $27: 16$ & $23: 39$ & $p=0.01$ \\
\hline Age (years) & $62.2 \pm 10.7$ & $64.3 \pm 11.2$ & $\mathrm{p}=0.3$ \\
\hline Duration of dialysis (months) & $33.7 \pm 33.5$ & $39.5 \pm 31.9$ & $\mathrm{p}=0.4$ \\
\hline Diagnosis, no. of cases (\%) & & & $p=0.9$ \\
\hline Glomerulonephritis & $11(25.6 \%)$ & $17(27.4 \%)$ & \\
\hline Diabetes & $17(39.5 \%)$ & $20(32.3 \%)$ & \\
\hline Hypertension & $4(9.3 \%)$ & $4(6.5 \%)$ & \\
\hline Polycystic & $1(2.3 \%)$ & $3(4.8 \%)$ & \\
\hline Obstruction & $1(2.3 \%)$ & $2(3.2 \%)$ & \\
\hline Others / unknown & $9(20.9 \%)$ & $16(25.9 \%)$ & \\
\hline \multicolumn{4}{|l|}{ Major comorbidity, no. of cases (\%) } \\
\hline Diabetes & $25(58.1 \%)$ & $30(48.4 \%)$ & $\mathrm{p}=0.3$ \\
\hline Coronary heart disease & $13(30.2 \%)$ & $16(25.8 \%)$ & $\mathrm{p}=0.6$ \\
\hline Cerebrovascular disease & $13(30.2 \%)$ & $21(33.9 \%)$ & $\mathrm{p}=0.7$ \\
\hline Charlson Index score & $6.5 \pm 1.9$ & $6.5 \pm 2.6$ & $p=0.9$ \\
\hline Machine assisted PD, no. of cases (\%) & $8(18.6 \%)$ & $5(8.1 \%)$ & $\mathrm{p}=0.1$ \\
\hline Previous peritonitis episodes* & $1(0-3)$ & $1(0-3)$ & $\mathrm{p}=0.9$ \\
\hline Species, no. of cases (\%) & & & $\mathrm{p}=0.5$ \\
\hline E. faecalis & $26(60.5 \%)$ & $32(51.6 \%)$ & \\
\hline E. faecium & $1(2.3 \%)$ & $10(16.1 \%)$ & \\
\hline others & 16 & $20(32.3 \%)$ & \\
\hline Type, no. of cases (\%) & & & $\mathrm{p}<0.0001$ \\
\hline isolated Enterococcus species & $25(58.1 \%)$ & $13(21.0 \%)$ & \\
\hline mixed bacterial growth & $18(41.9 \%)$ & $49(79.0 \%)$ & \\
\hline Adjuvant aminoglycoside, no. of cases (\%) & $9(20.9 \%)$ & $4(6.5 \%)$ & $p=0.03$ \\
\hline Ampicillin resistant, no. of cases (\%) & $5(11.6 \%)$ & $16(25.8 \%)$ & $\mathrm{p}=0.07$ \\
\hline
\end{tabular}

likely to have simple enterococcal peritonitis and had adjuvant aminoglycoside therapy (see Table 1).

The clinical outcome was summarized in Fig. 1 . The overall primary response rate to oral amoxicillin and IP vancomycin was $76.4 \%$ and $85.5 \%$, respectively ( $p=0.3$ ). The complete cure rate of oral amoxicillin and IP vancomycin was $55.8 \%$ and $54.8 \%$, respectively $(\mathrm{p}=0.8)$. Within the oral amoxicillin group, another 7 patients were cured by switching to IP vancomycin, giving an overall complete cure rate of $72.1 \%$. When the 5 episodes of ampicillin-resistant Enterococcus episodes were excluded, the primary response rate and complete cure rate of oral amoxicillin were $86.8 \%$ and $63.2 \%$, respectively. 


\section{Kidney Blood Pressure Research}

Kidney Blood Press Res 2017;42:837-843

\begin{tabular}{|l|l|l|l|l}
\hline DOI: 10.1159/000484426 & (C) 2017 The Author(s). Published by S. Karger AG, Basel
\end{tabular}

Published online: October 27, 2017

www.karger.com/kbr

\section{Discussion}

The optimal treatment of enterococcal peritonitis has been a controversial subject. Although clinical experience suggests that IP ampicillin is sometimes effective [7], there is always a concern that ampicillin has little in vitro activity when added to common PD solutions [11]. Routine treatment with IP vancomycin is currently recommended [10], but the possibility of provoking vancomycin-resistant strains is always a concern. Furthermore, there is evidence that vancomycin is less effective than ampicillin for Enterococcus that are penicillin-sensitive [15]. Since systemic administration of ampicillin or amoxicillin achieves therapeutic drug levels in the peritoneal cavity $[12,13]$, it is logical to expect that oral ampicillin or amoxicillin is effective for the treatment of enterococcal peritonitis. We favor amoxicillin over ampicillin because the former has better oral bioavailability [13] and requires only thrice daily dosage (as compared to four times per day for ampicillin).

According to the latest ISPD guideline, adjuvant IP aminoglycoside (usually gentamicin) could be used for Enterococcal peritonitis. In our present study, adjuvant aminoglycoside was used in only $12.4 \%$ cases. Because of the small number of case and possible selection bias, we did not perform subgroup analysis to explore the potential benefit of adjuvant aminoglycoside therapy. Nonetheless, oral amoxicillin offers another distinct advantage over IP ampicillin in this regard because aminoglycosides could not be added to the same bag of PD solution with ampicillin because of chemical incompatibility [16].

It is important to consider the local prevalence of ampicillin resistance before adopting the policy of oral amoxicillin treatment for enterococcal peritonitis. Notably, the prevalence of ampicillin resistance was $20 \%$ in our series, which may explain the good therapeutic response. In centers that have a high prevalence of ampicillin resistance, the efficacy of our regimen would be substantially inferior.

The optimal duration of treatment with oral amoxicillin remains undefined. According to the latest ISPD guideline, IP vancomycin for 3 weeks is required for enterococcal peritonitis [10]. In our present series, 32 patients had 2 weeks of oral amoxicillin, while 11 patients had 3 weeks of treatment. Because of the small sample size, we did not perform subgroup analysis to explore the effect of the duration of therapy. Nonetheless, the variable duration of treatment in our series may confound the interpretation of the result.

Enterococci are normal flora of the gastrointestinal tract [6]. Intra-abdominal pathology is generally considered to be the source of enterococcal peritonitis. Similar to previous reports [6,7], we found that other pathogenic organisms are isolated in over half of the enterococcal peritonitis episodes, and the coexistence of other organisms was associated with high rates of catheter removal, permanent hemodialysis transfer, and death. Our result suggests that oral amoxicillin, together with effective intra-peritoneal antibiotics for other concomitant organisms, is effective for polymicrobial peritonitis with Enterococcus as one of the causative organisms. From Fig. 1, it is possible that the response rate of polymicrobial peritonitis to oral amoxicillin is lower than simple enterococcal peritonitis, but the number of case was small and there are many confounding clinical factors that may affect the result.

Traditionally, identification of the exact species is important for enterococcal peritonitis because resistance to penicillin and carbapenems is far more frequently observed in $E$. faecium than E. faecalis [17]. In our present study, E. faecalis is the most common species identified. Amongst the 11 episodes caused by E. faecium, only one was treated with oral amoxicillin. Our result should therefore be extrapolated to this particular species with caution.

There are several inadequacies of our study. First, the sample size is small and the study is retrospective. As a result, treatment groups could not be compared without bias. For example, oral amoxicillin was used less often than vancomycin in those with mixed bacterial growth. There were more females in the vancomycin group than those in oral amoxicillin group. Adjuvant aminoglycoside was used less often in the vancomycin group than 


\section{Kidney \\ Blood Pressure Research}

amoxicillin group. In spite of the bias, our result suggests that oral amoxicillin is effective. We do not, however, prove that oral amoxicillin is superior (or not inferior) to IP vancomycin. Nonetheless, it seems unlikely to have randomized control trials in the future to compare IP vancomycin and oral amoxicillin, and our result suggests that the latter approach is a valid therapeutic option.

Second, we did not measure dialysate amoxicillin level to confirm it is well above the minimum bactericidal concentration. The dosage of amoxicillin that we used is similar to the recommended dosage for moderate infection in patients with normal kidney function, and should be considered relatively high for dialysis patients. This dosage was chosen in order to ensure sufficient drug level in the infected tissue. Unlike cephalosporins and carbapenems [18], high dose amoxicillin has minimal neurotoxicity and is generally well tolerated in dialysis patients [19]. On the other hand, neither did we measure serum or dialysate vancomycin level routinely. Although the dosage of IP vancomycin that we used was in accordance with the current guideline [10], it is possible that some patients, especially those with substantial residual renal function, might not have received an adequate dosage [20].

\section{Conclusion}

In summary, we found that oral amoxicillin has an excellent primary response rate and complete cure rate for PD related peritonitis episodes caused by Enterococcus species, especially when Enterococcus is the only bacteria isolated from the PD effluent. Our result indicates that oral amoxicillin is a valid and probably convenient therapeutic option for enterococcal peritonitis episodes.

\section{Disclosure Statement}

The authors declare no conflict of interests.

\section{Acknowledgements}

This study was supported by the Chinese University of Hong Kong (CUHK) research accounts 6901031 and 7101215. The funders had no role in study design, data collection and analysis, decision to publish, or preparation of the manuscript. Dr. CC Szeto receives research grant and consultancy from Baxter Healthcare. The results presented in this paper have not been published previously in whole or part, except in abstract format.

\section{References}

1 Mehrotra R, Devuyst O, Davies SJ, Johnson DW: The Current State of Peritoneal Dialysis. J Am Soc Nephrol 2016;27:3238-3252.

- Ho YW, Chau KF, Choy BY, Fung KS, Cheng YL, Kwan TH, Wong PN, Lai WM, Yuen SK, Lo SH, Chan CK, Leung CB: Hong Kong Renal Registry Report 2012 Hong Kong J Nephrol 2013;15: 28-43

3 Szeto CC, Chow KM, Wong TY, Leung CB, Wang AY, Lui SF, Li PK: Feasibility of resuming peritoneal dialysis after severe peritonitis and Tenckhoff catheter removal. J Am Soc Nephrol 2002;13:1040-1045.

4 Szeto CC, Leung CB, Chow KM, Kwan BC, Law MC, Wang AY, Lui SF, Li PK: Change in bacterial aetiology of peritoneal-dialysis-related peritonitis over ten years: experience from a center in South-East Asia. Clin Microbiol Infect 2005;10:837-839.

5 Wang Z, Jiang L, Feng S, Yang L, Jiang S, Zhan Z, Song K, Shen H: Early peritonitis is an independent risk factor for mortality in elderly peritoneal dialysis patients. Kidney Blood Press Res 2015;40:298-305. 


\section{Kidney \\ Blood Pressure Research}

-6 Edey M, Hawley CM, McDonald SP, Brown FG, Rosman JB, Wiggins KJ, Bannister KM, Johnson DW: Enterococcal peritonitis in Australian Peritoneal Dialysis Patients: Predictors, Treatment and Outcomes in 116 cases. Nephrol Dial Transplant 2010;25:1272-1278.

7 Yip T, Tse KC, Ng F, Hung I, Lam MF, Tang S, Lui SL, Lai KN, Chan TM, Lo WK: Clinical course and outcomes of single-organism Enterococcus peritonitis in peritoneal dialysis patients. Perit Dial Int 2011;31:522-528.

8 Sutherland SM1, Alexander SR, Feneberg R, Schaefer F, Warady BA: Enterococcal peritonitis in children receiving chronic peritoneal dialysis. Nephrol Dial Transplant 2010;25:4048-4054.

-9 Hollenbeck BL, Rice LB: Intrinsic and acquired resistance mechanisms in enterococcus: Virulence 2012;3:421-433.

10 Li PK, Szeto CC, Piraino B, de Arteaga J, Fan S, Figueiredo AE, Fish DN, Goffin E, Kim YL, Salzer W, Struijk DG, Teitelbaum I, Johnson DW: ISPD peritonitis recommendations: 2016 update on prevention and treatment. Perit Dial Int 2016;36:481-508.

11 Kussmann M, Schuster L, Zeitlinger M, Pichler P, Reznicek G, Wiesholzer M, Burgmann H, Poeppl W: The influence of different peritoneal dialysis fluids on the in vitro activity of ampicillin, daptomycin, and linezolid against Enterococcus faecalis. Eur J Clin Microbiol Infect Dis 2015;34:2257-2263.

12 Gerding DN, Hall WH, Schierl EA: Antibiotic concentrations in ascitic fluid of patients with ascites and bacterial peritonitis. Ann Intern Med 1977;86:708-713.

13 Geddes AM, Gould IM: Ampicillin, amoxicillin and other ampicillin-like penicillins. In: Grayson ML (eds). Kucers' The Use of Antibiotics: A Clinical Review of Antibacterial, Antifungal, Antiparasitic and Antiviral Drugs, 6th Edition. CRC Press, Florida, USA;2010, pp 65-92.

14 Szeto CC, Li PK, Johnson DW, Bernardini J, Dong J, Figueiredo AE, Ito Y, Kazancioglu R, Moraes T, van Esch S, Brown EA: ISPD catheter-related infections recommendations: 2017 update. Perit Dial Int 2017;37:141145 .

15 Abramowicz M: Choice of antibacterial drugs. Treat Guidel Med Lett 2007;5:33-50.

16 de Vin F, Rutherford P, Faict D: Intraperitoneal administration of drugs in peritoneal dialysis patients: a review of compatibility and guidance for clinical use. Perit Dial Int 2009;29:5-15.

17 Pérez-Fontán M, Rodríguez-Carmona A, Rodríguez-Mayo M: Enterococcal peritonitis in peritoneal dialysis patients: last name matters. Perit Dial Int 2011;31:513-517.

18 Chow KM, Szeto CC, Hui AC, Li PK: Mechanisms of antibiotic neurotoxicity in renal failure. Int J Antimicrob Agents 2004;23:213-217.

19 Chow KM, Hui AC, Szeto CC: Neurotoxicity induced by beta-lactam antibiotics: from bench to bedside. Eur J Clin Microbiol Infect Dis 2005;24:649-653.

20 Blunden M, Zeitlin D, Ashman N, Fan SL: Single UK centre experience on the treatment of PD peritonitisantibiotic levels and outcomes. Nephrol Dial Transplant 2007;22:1714-1719. 